\title{
Prevalence and origin of HIV-1 group M subtypes among patients attending a Belgian hospital in 1999
}

\author{
Joke Snoeck a, Sonia Van Dooren a , Kristel Van Laethem ${ }^{\text {a }}$, \\ Inge Derdelinckx ${ }^{\mathrm{a}, \mathrm{b}}$, Eric Van Wijngaerden ${ }^{\mathrm{b}}$, Erik De Clercq ${ }^{\mathrm{a}}$, \\ Anne-Mieke Vandamme ${ }^{\mathrm{a}, *}$ \\ ${ }^{a}$ Rega Institute for Medical Research, Katholieke Universiteit Leuven, Minderbroedersstraat 10, B-3000 Leuven, Belgium \\ ${ }^{\mathrm{b}}$ Internal Medicine, University Hospitals and Katholieke Universiteit Leuven, Leuven, Belgium
}

\begin{abstract}
HIV-1 group M strains are usually subtyped based on gag and/or env gene sequences. In our lab, part of the pol gene sequence was available in order to determine the genotypic anti-HIV drug resistance profile. To estimate the prevalence of the different HIV-1 subtypes in patients visiting the University Hospitals in Leuven in 1999 and for whom a genotypic drug resistance test was needed, we tried to use the pol sequence for subtyping. Recombination was investigated by similarity plots and bootscanning and subtyping was performed by phylogenetic analysis. The overall region spanning the entire protease and 747 nucleotides of the reverse transcriptase proved very suitable for subtyping, although there was a low phylogenetic signal at the beginning of the reverse transcriptase (nucleotides 0-250), as we demonstrated by likelihood mapping. Of the 41 samples analyzed, 21 belonged to subtype B. Of the other 20 non-B strains, 9 belonged to subtype C, 2 to subtype D and 1 to subtype A, G, H and J, respectively, 3 were CRF_02 (Circulating Recombinant Form), 1 was recombinant with a novel breakpoint and 1 sample was untypable. Although subtype B is still the most prevalent subtype in Belgium, it seems to be responsible for only half of the infections in this study. We could also document that the prevalence of subtype $\mathrm{C}$ is high in the Belgian native patients, especially among the heterosexually infected population. This could possibly be an indication for an epidemic spread of HIV-1 subtype C in Belgium, as for one third of these patients, no link to an endemic region could be found. The other non-B subtypes and the recombinants are mainly introduced by immigrants or by Belgian citizens traveling abroad. (C) 2002 Elsevier Science B.V. All rights reserved.
\end{abstract}

Keywords: Recombination breakpoint; Phylogenetic analysis; Circulating recombinant forms; Reverse transcriptase

\footnotetext{
* Corresponding author. Tel.: + 32-16-332160; fax: + 3216-332131.

E-mail address: annemie.vandamme@uz.kuleuven.ac.be (A.M. Vandamme).
}

\section{Introduction}

Human Immunodeficiency Virus (HIV), the causative agent of AIDS (Acquired Immune Deficiency Syndrome), has a big genomic diversity, caused mainly by the error-prone reverse transcriptase and the high replication rate of the virus. 
Recombination also plays an important role in the generation of sequence diversity by exchanging long fragments between different genomes. Two types of the virus exist, i.e. HIV-1 and HIV-2. HIV-1 can be classified into three large groups, based on the sequence information. Group $\mathrm{M}$ accounts for most of the HIV infections worldwide. The circulation of group $\mathrm{O}$ viruses seems to be restricted to West-Africa, where it is only responsible for less than $10 \%$ of the HIV-1 infections (Descamps et al., 1997). Recently a new group has been discovered in Cameroon, group $\mathrm{N}$ (Simon et al., 1998). HIV-2 infections, as HIV-1 group $\mathrm{O}$, remain largely confined to West-Central Africa (Esteves et al., 2000), although it has recently also been characterized in India and SouthKorea (Kannangai et al., 2000; Kim et al., 2000). HIV-1 group $M$ viruses can be subdivided into different subtypes according to their phylogeny, designated A-K (Korber et al., 2000). Each of the subtypes has a different geographic distribution (Paraskevis and Hatzakis, 1999). In Europe, North-America and Oceania subtype B is the most prevalent one, although there is evidence for the spread of non-B subtypes in these continents (Fransen et al., 1996; Barin et al., 1997; Dietrich et al., 1997; Boni et al., 1999; Balotta et al., 2001; Holguin et al., 2000; Weidle et al., 2000). As in other European countries, subtype B is most prevalent in Belgium, but a high prevalence of non-B subtypes has been reported before. Based on env data, Fransen et al. (1996) reported already in 1994 32\% non-B subtypes in Belgium.

Recently, there has been evidence that intersubtype recombinants are also taking part in the pandemic. Six so-called Circulating Recombinant Forms (CRF's) are recognized. These are recombinant strains that are taking part in the global epidemic, of which at least three epidemiologically unlinked sequences with the same breakpoint pattern are known (Salminen, 2000). Six such CRF's are currently recognized: CRF01_AE (Carr et al., 1996; Gao et al., 1996), CRF02_AG (Howard and Rasheed, 1996; Carr et al., 1998), CRF03 AB (Liitsola et al., 1998; Lukashov et al., 1999), CRF04_cpx (Gao et al., 1998; Nasioulas et al., 1999; Paraskevis et al., 2001), CRF05_DF (Laukkanen et al., 2000), and CRF06_cpx (Oelrichs et al., 1998; Montavon et al., 1999; Vidal et al., 2000). There are five other putative CRF's, but they are not generally accepted yet, because their epidemic spread still has to be confirmed (CRF07_BC, CRF08_BC, CRF09_cpx, CRF10_CD and CRF11_cpx). These recombinants may play a major role in local epidemics.

Mapping the distribution of subtypes and recombinants is important from an epidemiological point of view but also with respect to anti-HIV drug resistance and therapy response. Recombinants and pure subtypes may differ in all of these aspects, since differences between the subtypes have already been reported (Descamps et al., 1998; Apetrei et al., 1998). This distribution should also be considered in the development of vaccines and vaccine trials because a vaccine should include all the subtypes circulating in a certain area.

The subtype of new sequences is usually determined by sequencing and subsequent phylogenetic analysis. Other methods include the heteroduplex mobility assay (Heyndrickx et al., 2000) or the combinatorial melting assay (Kostrikis et al., 1998; Robbins et al., 1999). In general, the gag and/or env genes are used for subtyping, as these genes are more diverse than the pol gene, and therefore more appropriate for phylogenetic analysis of the closely related HIV-1 group M subtypes and their recombinant forms. However, the more conserved pol gene is sequenced in many labs to identify anti-HIV drug resistance-related mutations. As a consequence of current guidelines (Hirsch et al., 2000; Miller et al., 2001), many pol sequences will become available that could possibly be used for molecular epidemiological studies. The sequenced region in our lab, corresponding to nucleotides 2256 and 3329 of HXB2, is large enough to allow a reliable phylogenetic analysis with the purpose to identify the subtype. Using this region for molecular epidemiological purposes is thus appropriate, very cost-effective, and gives information concerning a large number of circulating HIV-1 strains in different countries.

In this study we used the viral protease gene and the $5^{\prime}$ part of the viral RT gene, obtained for the evaluation of the drug resistance profile, to investigate the prevalence of the HIV-1 group M 
subtypes in a Belgian hospital in 1999. Epidemiological analysis was performed to determine the origin of the non-B subtypes found.

\section{Materials and methods}

\subsection{Plasma samples}

In this study we included the pol sequences of virus strains from 41 patients who attended the University Hospitals in Leuven during 1999 and for whom a genotypic resistance analysis was performed. Fourteen patients were drug-experienced, failing their current regimen. The other 27 patients were drug-naive and were tested for baseline anti-HIV drug resistance-related mutations. The plasma viral load was determined using Quantiplex Branched DNA assay (Chiron Corporation, Emeryville, California) and varied between 1238 and 500000 copies $/ \mathrm{ml}$.

\section{2. $R N A$ extraction and $c D N A$ synthesis}

RNA extraction was performed using the $\mathrm{Nu}-$ clisens Lysis Kit and the Nuclisens Extraction Kit (Organon Teknika, Turnhout, Belgium), according to the protocol provided by the manufacturer. cDNA synthesis was done as previously described (Vandamme et al., 2000) by use of MuLV-RT (Applied Biosystems, Brussels, Belgium).

\subsection{Polymerase chain reaction}

Nested PCR was performed as previously described by Vandamme et al. (2000). The outer PCR amplified the protease and $5^{\prime}$ end of the reverse transcriptase with the primers AV150 and RT2. The inner PCRs were divided into the amplification of the protease gene with RVP5 and RVP3 and that of the $5^{\prime}$ end of the reverse transcriptase gene with primers M13USP-A35 and M13RSP-NE(1)35.

\subsection{Sequencing}

Sequencing was done on the ABI 310 or ABI 377 (Applied Biosystems) using the ABI Prism
dRhodamine Dye Terminator Cycle Sequencing Ready Reaction Kit or the ABI Prism BigDye Terminator Cycle Sequencing Ready Reaction Kit, according to the protocol provided by the manufacturer. The primers used for the protease gene were RVP5 and RVP3; those for the $5^{\prime}$ end of the reverse transcriptase gene were M13USP, M13RSP, AV36 and AV44, as described by Vandamme et al. (2000). Further analysis of the sequences was done using the Sequence Navigator software (Applied Biosystems).

\subsection{Phylogenetic analysis}

Sequences were aligned with reference sequences of RT and PRO group M subtypes available in the Los Alamos Database (http://www.ncbi.nlm.nih.gov/retroviruses) using CLUSTALW (Thompson et al., 1994), implemented in the Geneworks software package (Oxford Molecular Systems, Oxford, UK). The alignment was then edited using DAMBE (Xia, 2000). Gaps were removed manually and sequences were trimmed to equivalent length (958 nucleotides).

Simplot, version 2.5 , was used to generate similarity plots and bootscans. Simplot calculates and plots the percentage identity of the query sequence compared with a panel of reference sequences in a sliding window, which is moved across the alignment in steps (Ray, 1998). Bootscanning phylogeny involves tree construction with a chosen phylogenetic model and chosen parameters. We used the neighbor-joining method based on the Kimura two-parameter distance model with a transition/transversion bias of 2.0, after generation of 100 bootstrap replicates. Sequences were scored as pure subtypes or recombinants based on their pattern. Recombination breakpoints were determined visually by looking at the alignment and by a detailed bootscan of the query sequence and 3 reference subtypes, 2 of which would be the candidate subtypes for the recombination (Salminen et al., 1995). Usually, the recombination breakpoint could only be identified within a $20-50$ nucleotide region. The midpoint of this region was chosen as breakpoint. 
The subsequent detailed phylogenetic analyses were done using the PHYLIP software package, version 3.57 (Felsenstein, 1992). Evolutionary distances were calculated with DNADIST using the maximum likelihood nucleotide substitution model. The empirical determined transition/ transversion ratio of 2.8 was estimated in the MacClade program (Madison and Madison, 2000). Phylogenetic trees were generated with the neighbor-joining method and maximum likelihood method. Bootstrap support (1000 replicates) for the different clades of the NJ tree were calculated.

Analysis of the phylogenetic signal in different regions of the sequences was done using the likelihood mapping method, implemented in the software package Tree-Puzzle (Strimmer and von Haeseler, 1997). Saturation plots were made using DAMBE.

\section{Results}

\subsection{Patients characteristics}

Information on the patients was obtained by studying patients' files retrospectively. Seventyfour percent of the patients had the Belgian nationality. Of the remaining 26 percent, $8 \%$ was European and 18\% was African. Fourty-seven percent of the patients were probably infected in Belgium and these were all Belgians. Fourty-five percent were infected in Africa, $2 \%$ in SouthAmerica and the remaining $6 \%$ in the rest of Europe or the USA. More than half of the patients $(59 \%)$ became infected heterosexually, 36\% through homosexual contacts and 5\% due to intravenous drug use.

\subsection{Sequence information}

Only the samples for which pol sequences were obtained were included in this study; however, for two samples (p99-358 and p99-496), the inner PCR for the protease gene failed, and no protease sequence was obtained. For these samples, only the sequence of the reverse transcriptase could be included in the analyses. The sequenced region encompasses the entire protease and the $5^{\prime}$ part of the RT gene, codon 1-747. Sequences are available at the EMBL database under accession number AF338976 to AF339020.

\subsection{Subtyping of the strains}

First of all we wanted to determine the best window size and step size needed for the pol region sequenced to perform a confident subtyping using the Los Alamos webtool or Simplot subtyping and a confident determination of possible recombination breakpoints. Simplot was used for this purpose by taking a reference sequence of each subtype as query sequence and adjusting the window and step size to get the most accurate result. We got the best result with similarity plot and bootscan values above $80 \%$ using a window size of 400 and a step size of 20 nucleotides. These values were then used for subtyping with the Los Alamos webtool, and for similarity plotting and bootscanning using Simplot. The consensus value was set to $60 \%$, which means that the program constructs a consensus sequence for each subtype based on the nucleotides that are present in at least $60 \%$ of the reference strains used.

To acquire a rough idea about the subtype, the Los Alamos subtyping webtool was used (http:// www.ncbi.nlm.nih.gov/retroviruses). We maintained the CRF's in the reference strain list, although they caused dilution of the signal. For example, when a sequence is actually subtype B, CRF-06, which is also subtype B in a part of the considered region, will interfere possibly acquiring a higher percentage similarity with the query sequence as compared to the pure subtype B. It is however convenient to keep the CRF strains in this first rough subtyping, since this allows a fast recognition of real CRF strains.

The Simplot software package was used to obtain a similarity plot using Los Alamos reference strains, excluding the CRF's. Only when we suspected that a sequence was recombinant, we performed an additional analysis including the relevant CRF's to determine whether the sequence was a real CRF or a new recombinant. The results of these two methods were quite similar, as expected, since they both use similarity plotting to 
determine the subtype. The differences observed are mainly due to the interference of the CRF's which we included in the Los Alamos subtyping tool.

Bootscan analyses were done on all the sequences to identify possible recombinants and recombination breakpoints, using the same window size and step size as in the similarity plots.

Regions in the query sequence that did not reach the $75 \%$ level were assigned as untypable. Most results were unambiguous: only one sequence was clearly divergent (p99-154). Breakpoints were determined as described in Section 2. Three sequences showed the same pattern as the CRF_02. Two sequences had a breakpoint around nucleotide 505 of the reverse transcriptase, the first parts being $G$ and $D$, respectively; the second part $\mathrm{A}$ and $\mathrm{U}$ (untypable), respectively. However, phylogenetic analysis was more in favour of the pure subtypes $G$ and $D$. One sequence, a $\mathrm{C}+\mathrm{A}$ recombinant had a breakpoint around nucleotide 345 of the reverse transcriptase gene.

In our analysis we found that the first 250 nucleotides of the RT always gave a low phylogenetic signal. Saturation was checked with DAMBE by plotting the transitions and transversions against the genetic distance, but no saturation could be observed. Therefore, the amount of phylogenetic signal was analyzed with likelihood mapping. Fourty-two percent of the quartets were in the middle of the triangle, which means there was not enough phylogentic signal to perform reliable phylogenetic analysis, using only these 250 nucleotides. Therefore, care should be taken when drawing conclusions using this region.

To confirm the subtyping results, we performed phylogenetic analysis, using the PHYLIP software package as described in Section 2. The samples of which only the reverse transcriptase sequence was known, were analyzed separately (not shown). All clear non-recombinant sequences are shown in one tree (Fig. 1). Bootstrap values for all subtypes were above $95 \%$, except for subtype F1 (88\%) and subtype D $(76 \%)$. The recombinant sequences were divided into different parts according to the defined breakpoint and these parts were analyzed separately, after a preliminary analysis of the whole sequence. Fig. 2 shows the result of the analysis of one such recombinant (p99-589). Fig. 2a illustrates the analysis of the first part up to nucleotide 345 in the RT. This sequence clusters with subtype $\mathrm{C}$ with a bootstrap support of $80 \%$. The support for the subtype C-cluster without p99-589 was 91\%. Fig. 2b shows the NJ tree of the last part of the sequence. This part clusters with subtype A with a bootstrap value of $75 \%$. The A-cluster in this region without the query sequence is $100 \%$ supported. Fig. 2c shows the result of the bootscanning. Three CRF's number 2 (A/G recombinants) were identified. Including the CRF's in the tree construction, resulted in a CRF_02 cluster including our three recombinants with a bootstrap support of $100 \%$.

The results of the phylogenetic analysis confirm the bootscanning, both for the non-recombinant and the recombinant strains. Where the bootscanning identified two more recombinants, the phylogenetic analysis gave more support for the pure subtypes. The relevant second 242 nucleotide fragment of the RT gene, from RT nucleotide 505 to 747 , was, however, too short to perform a valuable analysis. In this region, the bootstrap support using only the reference strains for the different subtypes was indeed rather small, 11-100\%. For these two strains the bootstrap support to cluster the entire sequenced fragment with only a single subtype was very high, suggesting no solid support for them being recombinants.

We can conclude that most of the sequences (21) were subtype B; further, there were 9 subtype $\mathrm{C}$ strains (one of which was only known in RT), 2 subtype $\mathrm{D}$ strains, 1 subtype $\mathrm{A}, 1$ subtype $\mathrm{H}$ and 1 subtype $\mathrm{J}$ (RT-sequence) strain, respectively, 3 CRF_02 strains, $1 \mathrm{C} / \mathrm{A}$ recombinant with a novel breakpoint and 1 strain was untypable and may potentially be a new subtype (Fig. 3).

\subsection{Probable origin of the different subtypes found in our hospital}

Non-B subtypes and recombinants were present in persons from endemic regions in Africa or in Belgian patients with African partners from these regions $(49 \%)(1 \mathrm{H}, 2 \mathrm{D}$ and $1 \mathrm{G})$. None of the African patients were infected by subtype B 


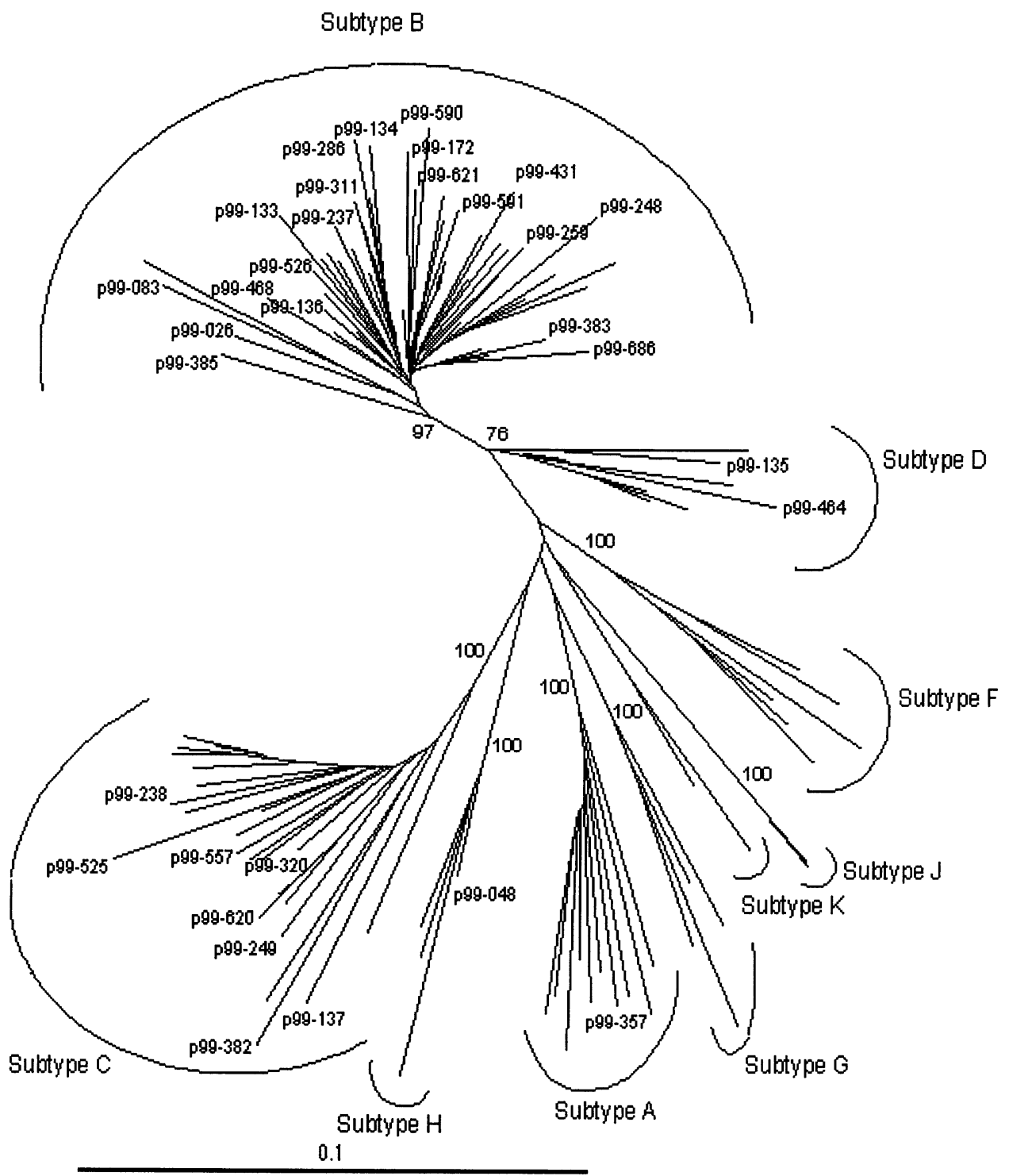

Fig. 1. Neighbor-Joining tree (constructed as indicated in Section 2), including all non-recombinant strains (indicated with a p99 number). HIV-1 group M subtypes A-K are indicated. Bootscanning support (1000 replicates) for the different subtypes is indicated in percentage on the branch leading to the subtype. Reference strains, starting clockwise, are: subtype B (AF069140, M17449, U37270, AF086817, U43141, K02007, M26727, AF004394, U34604, AF042100, U26546, U63632, K03455, U21135, D10112, AF070521, M38431, M93258, AF042101, U63632, AF042104, AF042103, AF042102, AF042105, M38429, U43096, U23487, M96155, U71182), subtype D (M22639, K03454, M27323, U88822, U88824), subtype F (F1: AJ249238, AF075703, AF077336, AF005494; F2: AJ249236, AJ249237), subtype J (AF082395, AF082394), subtype K (AJ249235, AJ249239), subtype G (AF061641, AF061642, AF084936, U88826, subtype A (AF069673, M62320, AF107771, U51190, AF004885, AF069672, AF069671, AF069670, AF069669), subtype H (AF190128, AF190127, AF005496) and subtype C (AF110959, AF110962, AF110967, U52953, AF110969, AF067154, AF067157, AF067159, AF067158, AF067155, AF110979, AF110978, AF110973, AF110972, U46016). 
viruses. Patients harbouring subtype B viruses had acquired the infection in countries where this subtype is most prevalent. This means that only subtype $\mathrm{C}$ was already circulating among the Belgian heterosexually infected patients in this hospital. We found that homosexual patients only harboured subtype B viruses and that probably all of them became infected in Belgium. No other subtypes were found in this population. Heterosexual patients harboured significantly more non-
B subtype viruses $(P<0.01)$ (Tables 1 and 2$)$. Within this heterosexually infected population, female patients carry more non-B subtypes than male patients $(P<0.01)$. No association could be found between the nationality of the patients and the subtype $(P=0.06)$, although there was a trend for non-Belgians carrying more non-B subtypes. There was, however, a clear association between the suspected origin of the virus and the subtype $(P<0.01)$ (Table 2).

(a)

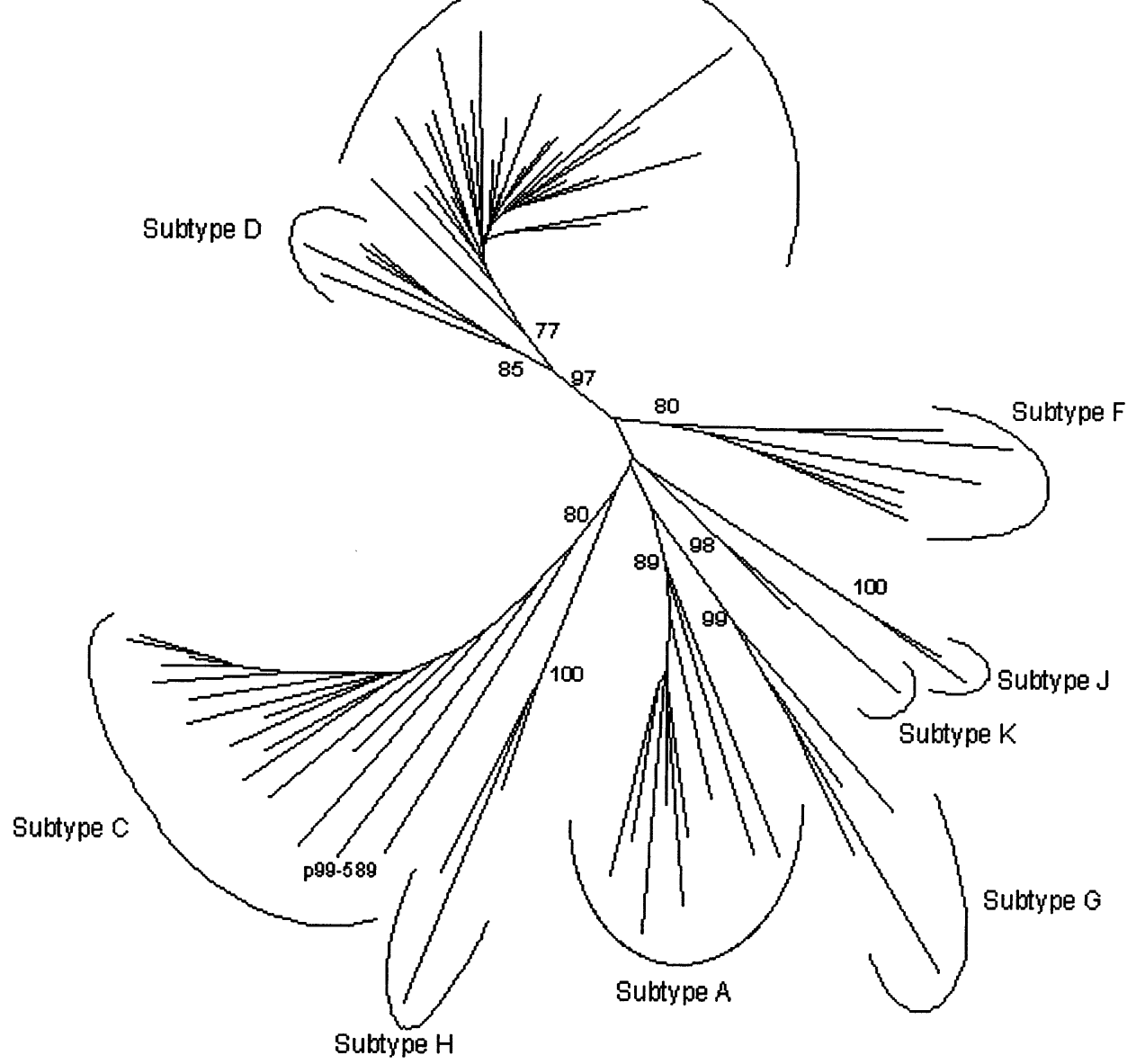

0.01

Fig. 2. Analysis of a recombinant sequence (p99-589). (a) The first part of the sequence (breakpoint at nucleotide 345 in RT) clusters with subtype $\mathrm{C}$ with a support of $80 \%$. (b) The last part of this sequence clusters with subtype A with a bootstrap value of $75 \%$. The same reference strains as in Fig. 1 were used. (c) Result of the bootscanning. The first part of the graphs clearly indicates subtype $\mathrm{C}$, and the second part indicates subtype A. 
(b)

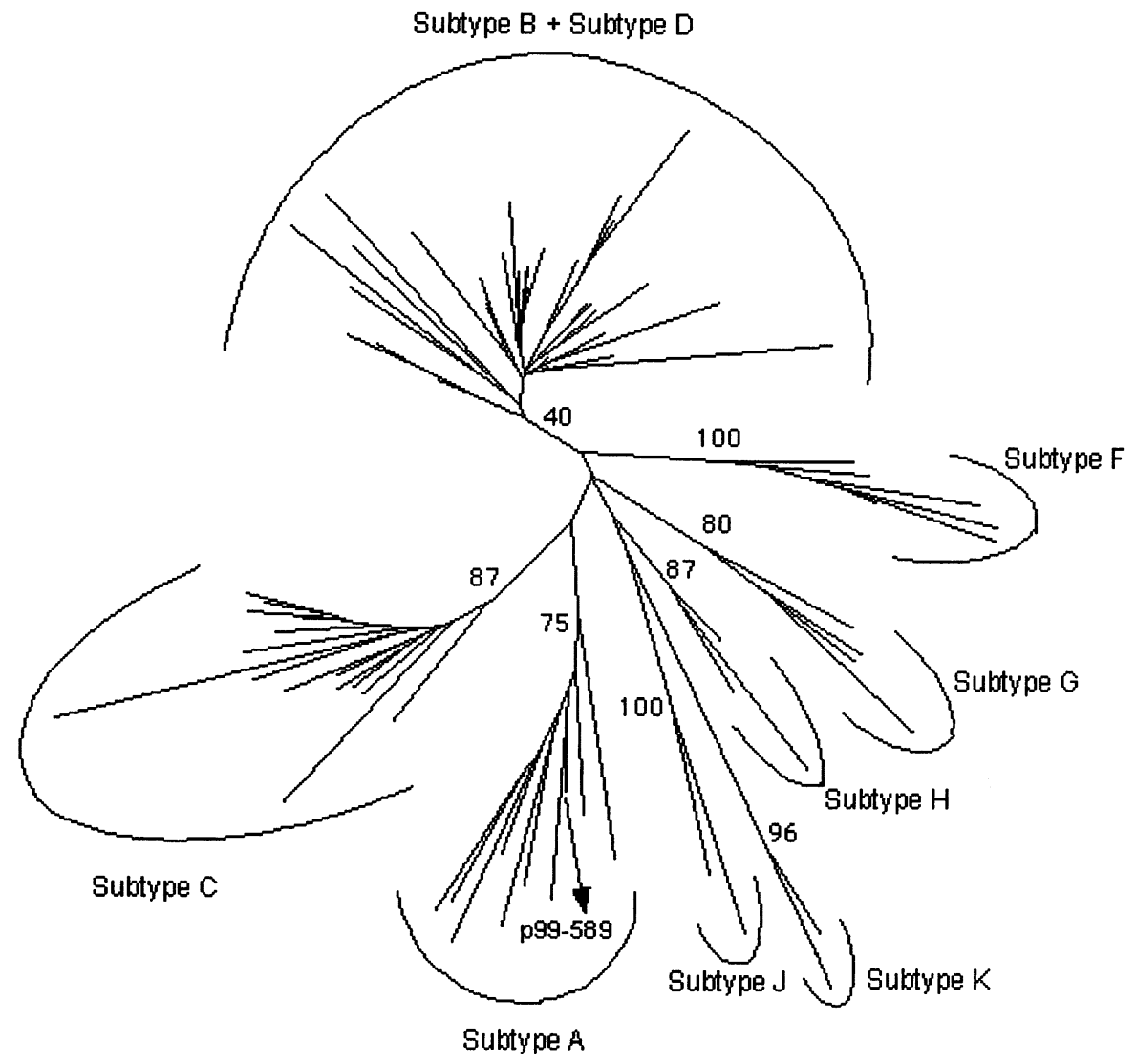

0.1

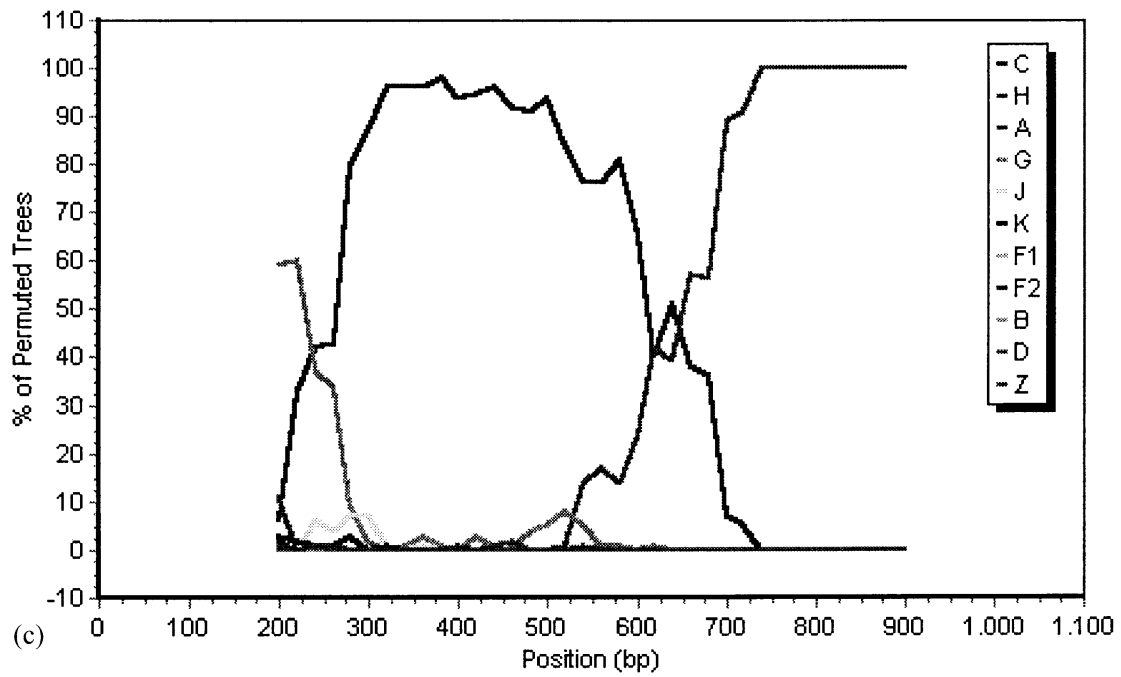

Window: 400 bn. Stev: 20 bn. GanStrin: On. Rens: 100 . Kirrura. Tt: 2.0 . NEIGHEOR

Fig. 2. (Continued) 


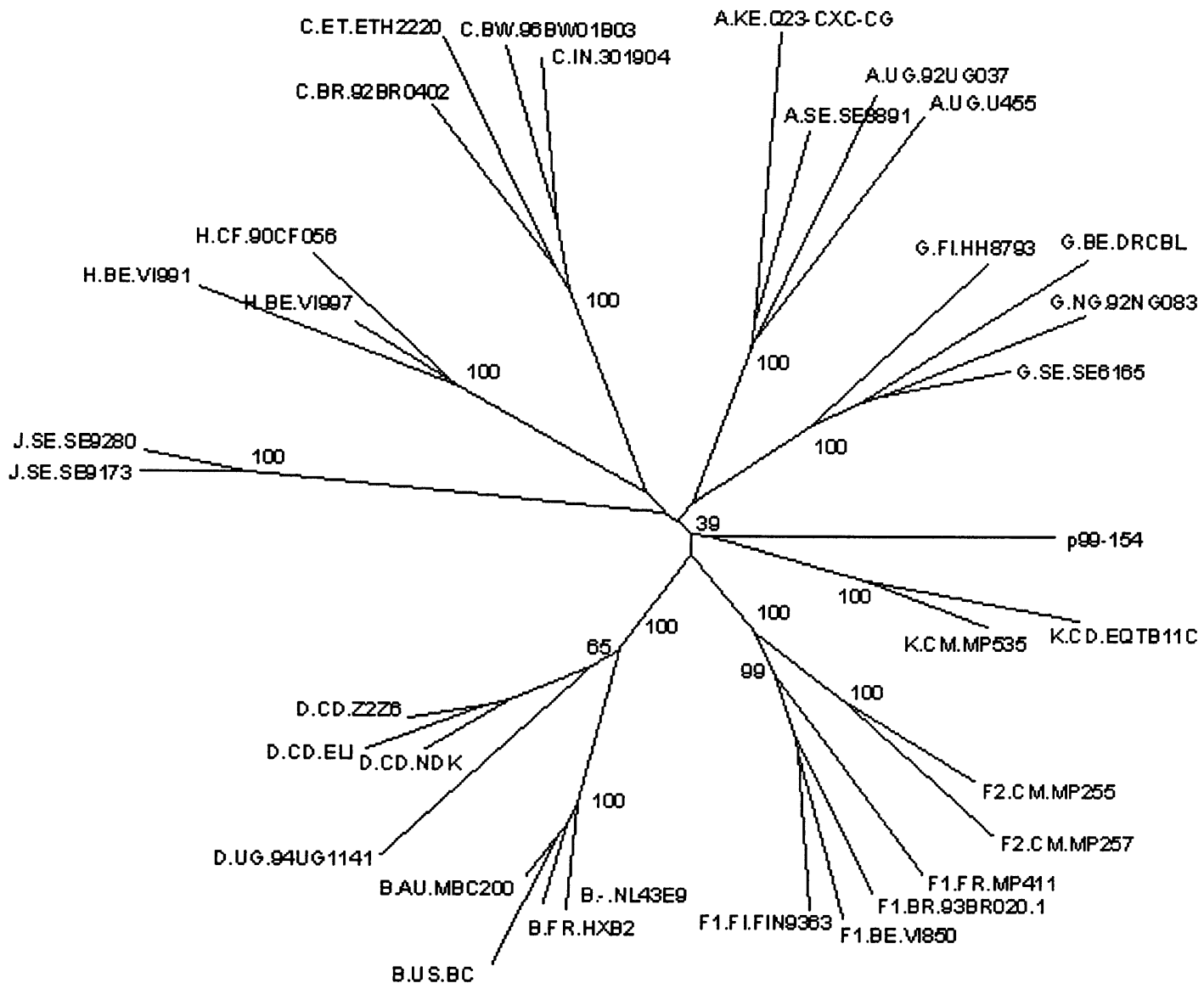

001

Fig. 3. Neighbor-Joining tree of the strain p99-154. There is no support for clustering this strain with any particular subtype.

\section{Discussion}

The HIV-1 pol gene is sequenced in many laboratories to determine the drug-resistance profile of the HIV strains. Following the recommendations to include resistance testing in routine clinical practice (Hirsch et al., 2000; Miller et al., 2001), many pol sequences will become available, that should be useful for molecular epidemiological studies. In the present study, we verified the suitability of this pol region (the entire protease and 747 nucleotides of the reverse transcriptase gene) for subtyping purposes, and tried to determine the origin of different subtypes identified in our hospital.
From our results, based on subtyping and retrospective data collection for 41 patients in our hospital, we could conclude that subtype B was still the most frequent subtype in 1999: 21 of 41 strains belonged to subtype B (51\%). As for the other subtypes, some are represented by a few cases; however, subtype $\mathrm{C}$ seems to be more prevalent within the investigated population. We also found one recombinant strain with a new breakpoint and one strain was untypable possibly representing a new subtype. In this study we also tried to determine the origin of the non-B subtypes and recombinants. We observed that people carrying subtype $\mathrm{B}$ virus acquired the infection from countries where subtype $B$ is the most preva- 
Table 1

Overview of the origin of the different subtypes found in our hospital in 1999

\begin{tabular}{|c|c|c|c|c|c|c|c|c|c|c|}
\hline & \multicolumn{10}{|c|}{ HIV subtype } \\
\hline & A & $\mathrm{B}$ & $\mathrm{C}$ & $\mathrm{D}$ & G & $\mathrm{H}$ & $\mathrm{J}$ & CRF_02 & $\mathrm{C} / \mathrm{A}$ & Divergent \\
\hline Total population (41) & 1 & 21 & 9 & 2 & 1 & 1 & 1 & 3 & 1 & 1 \\
\hline \multicolumn{11}{|l|}{ Heterosexual contacts (24) } \\
\hline African connection & 1 & 1 & 6 & 2 & 1 & 1 & 1 & 3 & 1 & 1 \\
\hline Belgian connection & & 1 & 3 & & & & & & & \\
\hline European/American connection & & 2 & & & & & & & & \\
\hline \multicolumn{11}{|l|}{ Homosexual contacts (15) } \\
\hline Belgian connection & & 15 & & & & & & & & \\
\hline \multicolumn{11}{|l|}{$I V D U(2)$} \\
\hline European/American connection & & 2 & & & & & & & & \\
\hline
\end{tabular}

lent subtype (Belgium, North America and Spain). Most of the people harbouring non-B subtypes were immigrants residing in Belgium and probably acquired the infection in their native country $(43 \%)$. Most of the Belgians infected with non-B subtypes, were probably infected outside Europe $(58 \%)$. This means that the recombinants, subtype $A, D, G, H$ and $J$ were therefore not yet circulating among the Belgian patients attending this hospital, but were introduced by immigrants or people who got infected in Africa. The picture for subtype $\mathrm{C}$ was different. One third of the people infected with this subtype are Belgians who got infected in Belgium. It is noteworthy that patients infected homosexually only harboured subtype B viruses, and that all of them probably were infected in Belgium. In contrast, no African patients carried a subtype B virus. The diversity of the subtypes circulating among the heterosexually infected population is larger, with significantly more female patients harbouring non-B subtypes than male heterosexuals. It should be noticed however that there were far more female non-Belgians than male non-Belgians in the study population. Patients infected with a virus through an African connection significantly harboured more non-B subtypes than patients who acquired the infection in Belgium.

In conclusion, it seems that bootscan combined with phylogenetic analysis is a reliable strategy for subtyping based on sequence information. Sub- type B was still the most prevalent subtype in Belgium, but it accounted for only half of the infections. Subtype B viruses were especially prevalent in the homosexually infected population. Most of the patients who harboured non-B subtypes were immigrants from endemic coun-

Table 2

Distribution of the HIV-1 group $\mathrm{M}$ subtypes within different categories

\begin{tabular}{|c|c|c|c|}
\hline & $\mathrm{B}$ & Non-B & $P$-value ${ }^{\mathrm{a}}$ \\
\hline \multicolumn{4}{|l|}{ Nationality } \\
\hline Belgian (30) & 18 & 12 & \\
\hline Non-Belgian (11) & 3 & 8 & 0.0655 \\
\hline \multicolumn{4}{|l|}{ Origin of the virus } \\
\hline Belgium (19) & 16 & 3 & \\
\hline Other (22) & 3 & 19 & 0.0004 \\
\hline \multicolumn{4}{|l|}{ Route of infection } \\
\hline Hetero (24) & 4 & 20 & $0.00004^{b}$ \\
\hline Homo (15) & 15 & 0 & \\
\hline IVDU' $^{c}(2)$ & 2 & 0 & \\
\hline \multicolumn{4}{|l|}{ Sex } \\
\hline Male (homo) (11) & 11 & 0 & \\
\hline Male (hetero) (12) & 6 & 6 & \\
\hline Female (14) & 2 & 12 & $0.00004^{\mathrm{d}}$ \\
\hline \multicolumn{4}{|c|}{$\begin{array}{l}{ }^{\text {a }} \text { Calculated using Fisher's exact test, a } P \text {-value }<0.05 \\
\text { considered significant, printed in bold. } \\
{ }^{b} \text { Comparing heterosexually infected with the others. } \\
{ }^{\mathrm{c}} \text { Intraveneous drug users. } \\
{ }^{\mathrm{d}} \text { Comparing male and female heterosexually infected pa } \\
\text { tients. }\end{array}$} \\
\hline
\end{tabular}


tries, except for a few Belgians who were infected abroad. HIV-1 subtype $\mathrm{C}$ however seems to have a relatively high prevalence among the Belgian native population $(26 \%)$. For one third of the subtype $\mathrm{C}$ viruses, no link to an endemic country could be found, possibly suggesting an epidemic spread of subtype $\mathrm{C}$ in Belgium. Care should be taken when interpreting this result, as it is based on only a limited amount of samples (9). Consequently, if this trend persists, the presence of subtype $\mathrm{C}$ in Belgium should be taken into account when studying vaccines, drug-resistance, epidemiology and therapy-response. In addition, the spread of the other subtypes should be carefully monitored.

\section{Acknowledgements}

The authors like to thank Marco Salemi and Phillipe Lemey for their assistance with the phylogenetic analysis. This work was partially supported by the Flemish Fonds voor Wetenschappelijk onderzoek (FWO-grant G.0288.01), the Belgian Geconcerteerde Onderzoeksacties (GOA 00/12) and the AIDS Reference Laboratory of Leuven which receives support from the Belgian Ministry of Social Affairs through a fund within the Health Insurance System.

\section{References}

Apetrei, C., Descamps, D., Collin, G., Loussert-Ajaka, I., Damond, F., Duca, M., Simon, F., Brun-Vézinet, F., 1998. Human immunodeficiency virus type 1 subtype $\mathrm{F}$ reverse transcriptase sequence and drug susceptibility. Journal of Virology 72 (5), 3534-3538.

Balotta, C., Facchi, G., Violin, M., Van Dooren, S., Cozzi-Lepri, A., Forbici, F., Bertoli, A., Riva, C., Senese, D., Caramello, P., Carnevale, G., Rizzardini, G., Cremonini, L., Monno, L., Rezza, G., Perno, C.F., Ippolito, G., d'Arminio-Monforte, A., Vandamme A.-M., Moroni, M., ICONA Study Group, 2001. Increasing prevalence of non-clade B HIV-1 strains in heterosexual men and women, as monitored by analysis of reverse transcriptase and protease sequences. Journal of Acquired Immune Deficiency Syndrome 27 (5), 499-505.

Barin, F., Courouce, A.M., Pillonel, J., Buzelay, L., 1997. Increasing diversity of HIV-1 M serotypes in French blood donors over a 10-year period (1985-1995). Retrovirus study group of the French society of blood transfusion. AIDS 11 (12), 1503-1508.

Boni, J., Pyra, H., Gebhardt, M., Perrin, L., Burgisser, P., Matter, L., Fierz, W., Erb, P., Piffaretti, J.C., Minder, E., Grob, P., Burckhardt, J.J., Zwahlen, M., Schupbach, J., 1999. High frequency of non-B subtypes in newly diagnosed HIV-1 infections in Switzerland. Journal of Acquired Immune Deficiency Syndrome 22 (2), 174-179.

Carr, J.K., Salminen, M.O., Koch, C., Gotte, D., Artenstein, A.W., Hegerich, P., St Louis, D., Burke, D.S., McCutchan, F.E., 1996. Full-length sequence and mosaic structure of a human immunodeficiency virus type 1 isolate from Thailand. Journal of Virology 70 (9), 5935-5943.

Carr, J.K., Salminen, M.O., Albert, J., Sanders-Buell, E., Gotte, D., Birx, D.L., McCutchan, F.E., 1998. Full genome sequences of human immunodeficiency virus type 1 subtypes $\mathrm{G}$ and $\mathrm{A} / \mathrm{G}$ intersubtype recombinants. Virology 247 (1), 22-31

Descamps, D., Collin, G., Letourneur, F., Apetrei, C., Damond, F., Loussert-Ajaka, I., Simon, F., Saragosti, S., Brun-Vézinet, F., 1997. Susceptibility of human immunodeficiency virus type 1 group $\mathrm{O}$ isolates to antiretroviral agents: in vitro phenotypic and genotypic analyses. Journal of Virology 71 (11), 8893-8898.

Descamps, D., Apetrei, C., Collin, G., Damond, F., Simon, F., Brun-Vézinet, F., 1998. Naturally occurring decreased susceptibility of HIV-1 subtype G to protease inhibitors. AIDS 12 (9), 1109-1111.

Dietrich, U., Ruppach, H., Gehring, S., Knechten, H., Knickmann, M., Jager, H., Wolf, E., Husak, R., Orfanos, C.E., Breda, H.D., Rubsamen-Waigmann, H., von Briesen, H., 1997. Large proportion of non-B HIV-1 subtypes and presence of zidovudine resistance mutations among German seroconvertors. AIDS 11 (12), 1532-1533.

Esteves, A., Parreira, R., Piedade, J., Venenno, T., Canas-Ferreira, W.F., 2000. Genetic characterization of HIV type 1 and type 2 from Bissau, Guinea-Bissau (West Africa). Virus Research 68 (1), 51-61.

Felsenstein, J., 1992. PHYLIP (Phylogeny Inference Program), $3.5 \mathrm{c}$ ed. Department of Genetics, University of Washington, Seattle, Washington.

Fransen, K., Buvé, A., Nkengasong, J.N., Laga, M., van der Groen, G., 1996. Longstanding presence in Belgians of multiple non-B HIV-1 subtypes. Lancet 347 (9012), 1403.

Gao, F., Robertson, D.L., Morrison, S.G., Hui, H., Craig, S., Decker, J., Fultz, P.N., Girard, M., Shaw, G.M., Hahn, B.H., Sharp, P.M., 1996. The heterosexual human immunodeficiency virus type 1 epidemic in Thailand is caused by an intersubtype $(\mathrm{A} / \mathrm{E})$ recombinant of African origin. Journal of Virology 70 (10), 7013-7029.

Gao, F., Robertson, D.L., Carruthers, C.D., Li, Y., Bailes, E., Kostrikis, L.G., Salminen, M.O., Bibollet-Ruche, F., Peeters, M., Ho, D.D., Shaw, G.M., Sharp, P.M., Hahn, B.H., 1998. An isolate of human immunodeficiency virus type 1 originally classified as subtype I represents a complex mosaic comprising three different group M subtypes (A, G, and 1). Journal of Virology 72 (12), 10234-10241. 
Heyndrickx, L., Janssens, W., Zekeng, L., Musonda, R., Anagonou, S., Van der Auwera, G., Coppens, S., Vereecken, K., De Witte, K., Van Rampelbergh, R., Kahindo, M., Morison, L., McCutchan, F.E., Carr, J.K., Albert, J., Essex, M., Goudsmit, J., Asjo, B., Salminen, M., Buve, A., van der Groen, G., 2000. Simplified strategy for detection of recombinant human immunodeficiency virus type 1 group $\mathrm{M}$ isolates by gag/env heteroduplex mobility assay. Study group on heterogeneity of HIV epidemics in African cities. Journal of Virology 74 (1), $363-370$.

Hirsch, M.S., Brun-Vezinet, F., D’Aquila, R.T., Hammer, S.M., Johnson, V.A., Kuritzkes, D.R., Loveday, C., Mellors, J.W., Clotet, B., Conway, B., Demeter, L.M., Vella, S., Jacobsen, D.M., Richman, D.D., 2000. Antiretroviral drug resistance testing in adult HIV-1 infection: recommendations of an International AIDS Society-USA Panel. Journal of the American Medical Association 283 (18), 2417-2426.

Holguin, A., Rodes, B., Soriano, V., 2000. Protease gene analysis of HIV type 1 non-B subtypes in Spain. AIDS Research and Human Retroviruses 16 (14), 1395-1403.

Howard, T.M., Rasheed, S., 1996. Genomic structure and nucleotide sequence analysis of a new HIV type 1 subtype A strain from Nigeria. AIDS Research and Human Retroviruses 12 (15), 1413-1425.

HIV-1 subtypes: implications for epidemiology, pathogenicity, vaccines and diagnostics. Workshop Report from the European Commission (DG XII, INCO-DC) and the Joint United Nations Programme on HIV/AIDS (1997). AIDS 11 (15), UNAIDS17-UNAIDS36.

Kannangai, R., Ramalingam, S., Prakash, K.J., Abraham, O.C., George, R., Castillo, R.C., Schwartz, D.H., Jesudason, M.V., Sridharan, G., 2000. Molecular confirmation of human immunodeficiency virus (HIV) type 2 in HIVseropositive subjects in South-India. Clinical and Diagnostic Laboratory Immunology 7 (6), 987-989.

Kim, S.S., Kim, E.Y., Park, K.Y., Suh, S.D., Park, H.K., Shin, Y.O., Bae, M., Lee, J.S., 2000. Introduction of human immunodeficiency virus 2 infection into South-Korea. Acta Virologica 44 (1), 15-22.

Korber, B., Hahn, B., Foley, B., Mellors, J.W., Leitner, T., Myers, G., McCutchan, F., Kuiken, C., 2000. Human Retroviruses and AIDS 1997: A Compilation and Analysis of Nucleic Acid and Amino Acid Sequences. Los Alamos National Laboratory, Los Alamos, New Mexico.

Kostrikis, L.G., Shin, S., Ho, D.D., 1998. Genotyping HIV-1 and HCV strains by a combinatorial DNA melting assay (COMA). Molecular Medicine 4 (7), 443-453.

Laukkanen, T., Carr, J.K., Janssens, W., Liitsola, K., Gotte, D., McCutchan, F.E., Op de Coul, E., Cornelissen, M., Heyndrickx, L., van der Groen, G., Salminen, M.O., 2000. Virtually full-length subtype $\mathrm{F}$ and F/D recombinant HIV1 from Africa and South America. Virology 269 (1), 95 104.

Liitsola, K., Tashkinova, I., Laukkanen, T., Korovina, G., Smolskaja, T., Momot, O., Mashkilleyson, N., Chaplin- skas, S., Brummer-Korvenkontio, H., Vanhatalo, J., Leinikki, P., Salminen, M.O., 1998. HIV-1 genetic subtype A/B recombinant strain causing an explosive epidemic in injecting drug users in Kaliningrad. AIDS 12 (14), 19071919.

Lukashov, V.V., Huismans, R., Rakhmanova, A.G., Lisitsina, Z.N., Akhtyrskaya, N.A., Vlasov, N.N., Melnick, O.B., Goudsmit, J., 1999. Circulation of subtype A and gagA/ $e n v \mathrm{~B}$ recombinant HIV type 1 strains among injecting drug users in St. Petersburg, Russia, correlates with geographical origin of infections. AIDS Research and Human Retroviruses 15 (17), 1577-1583.

Madison, D.R., Madison, W.P., MacClade, version 4.0, distributed by the authors. Available from http:// phylogeny.arizona.edu/macclade/macclade.html.

Miller, V., Vandamme, A.-M., Loveday, C., Staszewski, S., Lundgren, J., Youle, M., Ait-Khaled, M., Boucher, C., Brun-Vézinet, F., Dedes, N., Giaquinto, C., Hertogs, K., Houyez, F., Perrin, L., Pillay, D., Schmit, J.-C., Schuurman, R., Lange, J., 2001. Clinical and laboratory guidelines for the use of HIV-1 drug resistance testing as part of treatment management-recommendations for the European setting. AIDS 15, 309-320.

Montavon, C., Bibollet-Ruche, F., Robertson, D., Koumare, B., Mulanga, C., Esu-Williams, E., Toure, C., Mboup, S., Saman, E., Delaporte, E., Peeters, M., 1999. The identification of a complex $\mathrm{A} / \mathrm{G} / \mathrm{I} / \mathrm{J}$ recombinant HIV type I virus in various West African countries. AIDS Research and Human Retroviruses 15 (18), 1707-1712.

Nasioulas, G., Paraskevis, D., Magiorkinis, E., Theodoridou, M., Hatzakis, A., 1999. Molecular analysis of the fulllength genome of HIV type 1 subtype I: evidence of A/G/I recombination. AIDS Research and Human Retroviruses 15 (8), 745-758.

Oelrichs, R.B., Workman, C., Laukkanen, T., McCutchan, F.E., Deacon, N.J., 1998. A novel subtype A/G/J recombinant full-length HIV type 1 genome from Burkina Faso. AIDS Research and Human Retroviruses 14 (16), 1495 1500 .

Paraskevis, D., Hatzakis, A., 1999. Molecular epidemiology of HIV-1 infection. AIDS Reviews 1, 238-249.

Paraskevis, D., Magiorkinis, E., Magiorkinis, G., Anastassopoulou, C., Lazanas, M., Chrysos, G., Vandamme, A.-M., Hatzakis, A., 2001. Molecular characterization of a complex recombinant human immunodeficiency virus type 1 (HIV-1) isolate $(\mathrm{A} / \mathrm{G} / \mathrm{J} / \mathrm{K} /$ ?): evidence to support the existence of a novel HIV-1 subtype. Journal of General Virology 82 (Pt 10), 2509-2514.

Ray, S.C., 1998. Simplot for Windows (version 1.6). Baltimore, Maryland: distributed by author. Available from http://www.med.ihu.edu/deptmed/sray/download.

Robbins, K.E., Kostrikis, L.G., Brown, T.M., Anzala, O., Shin, S., Plummer, F.A., Kalish, M.L., 1999. Genetic analysis of human immunodeficiency virus type I strains in Kenya: a comparison using phylogenetic analysis and a combinatorial melting assay. AIDS Research and Human Retroviruses 15 (4), 329-335. 
Salminen, M.O., Carr, J.K., Burke, D.S., McCutchan, F.E., 1995. Identification of breakpoints in intergenotypic recombinants of HIV type 1 by bootscanning. AIDS Research and Human Retroviruses 11 (11), 1423-1425.

Salminen, M.O., 2000. HIV inter-subtype recombinationconsequences for the epidemic. AIDS Reviews 2 (3), 178 189.

Simon, F., Loussert-Ajaka, I., Muller-Trutwin, M.C., Saragosti, S., Georges-Courbot, M.C., Barré-Simoussi, F., Brun-Vézinet, F., 1998. Identification of a new human immunodeficiency virus type 1 distinct from group $\mathbf{M}$ and group O. Nature Medicine 4 (9), 1032-1037.

Strimmer, K, von Haeseler, A., 1997. Likelihood-mapping: a simple method to visualize phylogenetic content of a sequence alignment. Proceedings of the National Academy of Sciences of the USA 94, 6815-6819.

Thompson, J.D., Higgins, D.G., Gibson, T.J., 1994. CLUSTAL W: improving the sensitivity of progressive multiple sequence alignment through sequence weighting, position-specific gap penalties and weight matrix choice. Nucleic Acids Research 22 (22), 4673-4680.

Vandamme, A.-M., Witvrouw, M., Pannecouque, C., Balzarini, J., Van Laethem, K., Schmit, J.-C., Desmyter, J.,
De Clercq, E., 2000. Evaluating clinical isolates for their phenotypic and genotypic resistance against anti-HIV drugs. In: Kinchington, D., Schinazi, R.F. (Eds.), Methods in Molecular Medicine. Humana Press Inc, Totowa, New York, pp. 223-258.

Vidal, N., Mulanga-Kabeya, C., Nzilambi, N., Delaporte, E., Peeters, M., 2000. Identification of a complex env subtype E HIV type 1 virus from the Democratic Republic of Congo, recombinant with $\mathrm{A}, \mathrm{G}, \mathrm{H}, \mathrm{J}, \mathrm{K}$, and unknown subtypes. AIDS Research and Human Retroviruses 16 (18), 2059-2064.

Weidle, P.J., Ganea, C.E., Irwin, K.L., Pieniazek, D., McGowan, J.P., Olivo, N., Ramos, A., Schable, C., Lal, R.B., Holmberg, S.D., Ernst, J.A., 2000. Presence of human immunodeficiency virus (HIV) type 1, group M, nonB subtypes, Bronx, New York: a sentinel site for monitoring HIV genetic diversity in the United States. Journal of Infectious Diseases 181 (2), 470-475.

Xia, X., 2000. DAMBE (Data analysis in molecular biology and evolution). Department of Ecology and Biodiversity, University of Hong Kong; distributed by author. Available from http://web.hku.hk/ xxia/software/software.htm. 